Henrique Martins Galvão, Rosinei Batista Ribeiro, Wellington de Oliveira *

\title{
Design, Reflexões e Experiências: contribuições de um Programa de Mestrado Profissional para 0 avanço do conhecimento em Design
}

Henrique Martins Galvão possui doutorado em Administração pela FEA-USP), exercendo a função de Coordenador do PPG lato-sensu e stricto-sensu no Mestrado Profissional em Design, Tecnologia e Inovação (PPG-DTI). Possui publicações em periódicos e congressos que compreendem os seguintes temas: Administração Financeira, Gestão Estratégica, Inovação Tecnológica Ambiental, Eco-inovação, Responsabilidade Socioambiental Empresarial, Marketing, Logística e Cadeia de Suprimentos, Empreendedorismo e Gestão Acadêmica. <galvaohm@gmail.com> ORCID: 0000-0002-8017-6537
Resumo experiências que dizem respeito às atividades de ensino, pesquisa e de extensão do Programa de Mestrado Profissional em Design, Tecnologia e Inovação, do Centro Universitário Teresa D'Ávila - UNIFATEA, na cidade Lorena, no estado de São Paulo, sendo considerado para contextualização o recorte do período compreendido entre os anos de 2015 a 2019. No âmbito do processo avaliativo de programas stricto sensu conduzido pela Coordenação de Aperfeiçoamento de Pessoal de Nível Superior - CAPES, constata-se o significativo papel desempenhado pelo planejamento. Desse modo, aponta-se que o planejamentos dos PPGs perpassa pela Interdisciplinaridade, Internacionalização e a Interinstitucionalização como elementos chave e estratégicos na articulação dos projetos desenvolvidos na Área do Conhecimento. Portanto, no PPG-DTI, esse processo é amparado por meio do alinhamento com a missão, visão e valores do UNIFATEA, instituição confessional católica salesiana, bem como as características socioeconômicas geográficas e pelo estabelecimento de convênios institucionais (nacional e internacional), de modo a compartilhar ensino, a pesquisa e o desenvolvimento, a transferência e a difusão do conhecimento e tecnologia por meio de laboratórios próprios ou da própria IES e/ou empresas conveniadas para pesquisa aplicada.

Palavras chave Design, Tecnologia, Inovação, Conhecimento. 


\section{Design, Reflections and Experiences: contributions of a Professional Master's Pro- gram to the advancement of knowledge in Design}

Rosinei Batista Ribeiro é Pós-Doutorado em Engenharia de Materiais no Instituto Tecnológico de Aeronáutica (ITA). Doutorado e Mestrado em Engenharia Mecânica pela Universidade Estadual Paulista Júlio de Mesquita Filho (FEG-UNESP). Pró-Reitor de Pesquisa, Pós-Graduação e Extensão e Docente Permanente no Programa de Mestrado Profissional em Design, Tecnologia e Inovação no Centro Universitário Teresa D’Ávila (UNIFATEA). Líder do Grupo de Pesquisa "Projeto de Produto e Tecnologias Sociais", registrado no Diretório de Grupos de Pesquisa do CNPq. Docente Permanente no Programa de Mestrado Profissional em Gestão e Tecnologia em Sistemas Produtivos no Centro Estadual de Educação Tecnológica Paula Souza (CEETEPS).<rosinei1971@gmail.com > ORCID: 0000-0001-8225-7819

Wellington de Oliveira é Doutor e pós-doutor pela Pontifícia Universidade Católica de São Paulo. Professor permanente do Programa de estudos pós-graduados em Design, Inovação e Tecnologia no Centro Universitário Teresa D’Ávila- UNIFATEA e coordenador do LAPED- Laboratório de Pesquisas em Design e Educação na mesma instituição. <reitoria@fatea.br> ORCID: 0000-0003-0523-5645
Abstract This article aims to contextualize the practices that converge the experiences related to the teaching, research and extension activities of the Professional Master's Program in Design, Technology and Innovation, at the Teresa D'Ávila University Center - UNIFATEA, in the city Lorena, in the state of São Paulo, taking into account the context of the period from 2015 to 2019 for context. Within the scope of the evaluation process of stricto sensu programs conducted by the Coordination for the Improvement of Higher Education Personnel - CAPES, the significant role played by planning. Thus, it is pointed out that the planning of PPGs goes through Interdisciplinarity, Internationalization and Interinstitutionalization as key and strategic elements in the articulation of projects developed in the Area of Knowledge. Therefore, in the PPG-DTI, this process is supported through alignment with the mission, vision and values of UNIFATEA, a Salesian Catholic confessional institution, as well as the geographical socioeconomic characteristics and the establishment of institutional agreements (national and international), in a way to share teaching, research and development, the transfer and diffusion of knowledge and technology through its own laboratories or the HEI itself and / or companies associated with applied research.

Keywords Design, Technology, Innovation, Knowledge.

Diseño, Reflexiones y Experiencias: contribuciones de una Maestría Profesional al avance del conocimiento en Diseño

Resumen Este artículo tiene como objetivo contextualizar las prácticas que confluyen las experiencias relacionadas con las actividades de docencia, investigación y extensión del Programa de Maestría Profesional en Diseño, Tecnología e Innovación, en el Centro Universitario Teresa D'Ávila - UNIFATEA, en la ciudad Lorena, en el estado de São Paulo, teniendo en cuenta el contexto del período de 2015 a 2019 para el contexto. En el ámbito del proceso de evaluación de los programas stricto sensu realizado por la Coordinación de Perfeccionamiento del Personal de Educación Superior-CAPES, el importante papel desempeñado por planificación. Así, se señala que la planificación de los PPG pasa por la Interdisciplinariedad, Internacionalización e Interinstitucionalización como elementos clave y estratégicos en la articulación de proyectos desarrollados en el Área del Conocimiento. Por tanto, en el PPG-DTI, este proceso se sustenta a través de la alineación con la misión, visión y valores de UNIFATEA, institución confesional católica salesiana, así como las características geográficas socioeconómicas y el establecimiento de acuerdos institucionales (nacionales e internacionales). , de manera de compartir la docencia, la investigación y el desarrollo, la transferencia y difusión del conocimiento y la tecnología a través de sus propios laboratorios o de la propia IES y / o empresas asociadas a la investigación aplicada.

Palabras clave Diseño, Tecnología, Innovación, Conocimiento. 


\section{Introdução}

O presente artigo conduz à oportunidade de apresentar o Programa de Mestrado Profissional em Design, Tecnologia e Inovação do Centro Universitário Teresa D'Ávila - UNIFATEA - PPG-DTI, a partir do seu protagonismo local, regional. As contribuições do PPG-DTI são oriundas das suas ações e práticas para o fortalecimento da Área de Arquitetura, Urbanismo e Design. Em especial, destaca-se a inserção do Programa na subárea do Design, tendo como condição permanente a interlocução do conhecimento com outras áreas. O Programa encontra-se alicerçado na área de concentração em design, tecnologia e inovação, norteado por duas linhas de pesquisa, a saber: projeto de produto e inovação e empreendedorismo. Nas interfaces dos dois eixos, busca-se a indissociabilidade entre o ensino, a pesquisa e a extensão, e que respondem à produção de conhecimentos valorizados, principalmente, pela abordagem interdisciplinar e multidisciplinar do exercício do design. Para cada linha de pesquisa do PPG-DTI associam-se aos projetos de pesquisa do docentes, os quais podem ser compartilhas por ambas linhas de pesquisa a partir dos seus projetos.

Fig 1. articulação linhas de pesquisas e projetos Fonte: PPG-DTI (2020)

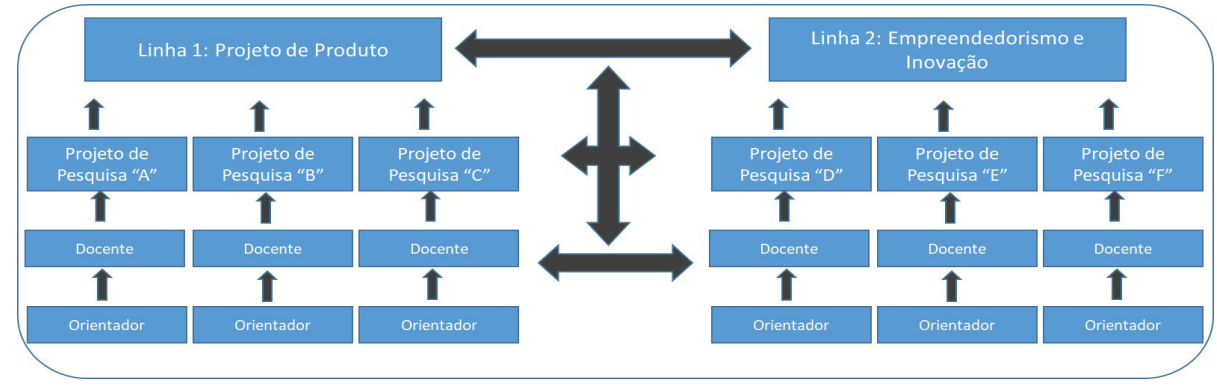

Posto que a área de concentração do PPG-DTI expressa a sua vocação para a produção de conhecimento sob o domínio das linhas de pesquisa. Para tanto, a legitimação leva em conta o atendimento dos pressupostos dos objetivos e de formação de egressos amparadas pela sua estrutura curricular, sendo constituída pelo conjunto de 17 disciplinas, obrigatórias e eletivas, com carga horária de 60 horas e 4 créditos cada. As unidades curriculares obrigatórias se subdividem em: Design e Tecnologia; Design e Transferência de Tecnologia; Metodologia da Pesquisa e Seminários Avançados em Design. As disciplinas eletivas se constituem em: Desenvolvimento, Tecnologia e Inovação; Design de Superfície e Modelagem; Design Thinking; Ecodesign; Ergonomia; Estatística Aplicada ao Design; Gestão da Produção e Operações; Inovação e Aspectos Cognitivos; Inovação Industrial; Práticas corporativas e Empreendedorismo; Práticas de Prototipagem; Processos em Indústrias Criativas e Desenvolvimento Regional; Seleção de Materiais e Processos de Fabricação e Tópicos avançados: Design, Subjetividade e Mídias Digitais Interativas. 
Desde março de 2015, quando o UNIFATEA obteve a homologação do projeto pelo CTC-ES/CAPES, e conferido o reconhecimento conforme o Parecer CNE/CES nํ. 46/2016, publicado pela Portaria nº. 919, do Ministério da Educação, de 18 de agosto de 2016, foram concluídas 65 dissertações, no período de 2015 a 2019. - PPT's gerados por linha de pesquisa Fonte: PPG-DTI (2020)
Fig 2. Produtos técnico-tecnológicos

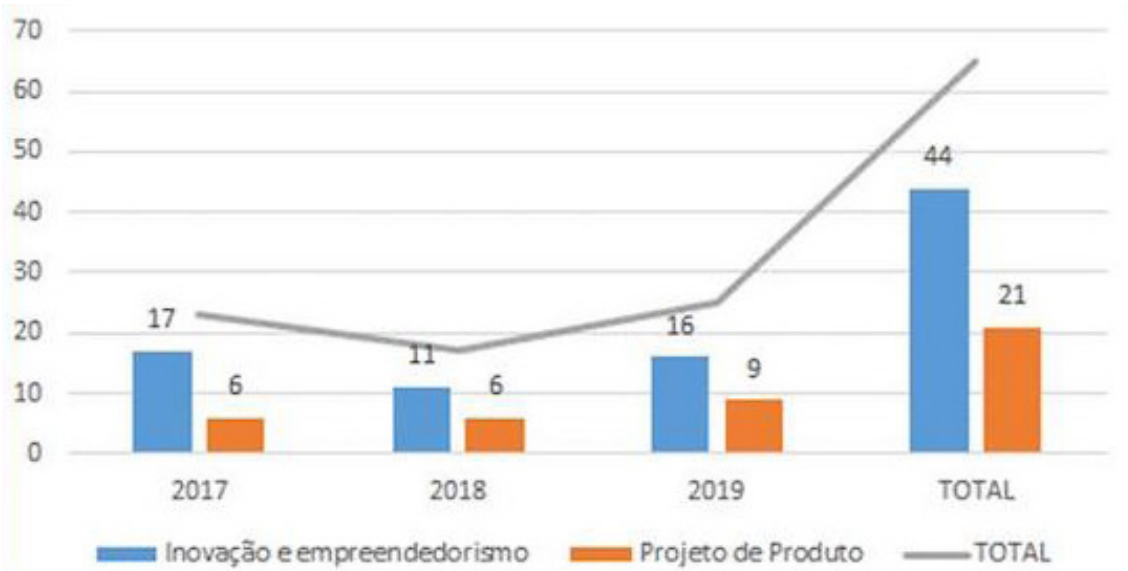

A tipificação dos produtos técnico-tecnológicos permitiu extrair a classificação das produções no período e que se compuseram $56 \%$ na Linha de Pesquisa em Projeto de Produto e $44 \%$ na Linha de Pesquisa em Inovação e Empreendedorismo.

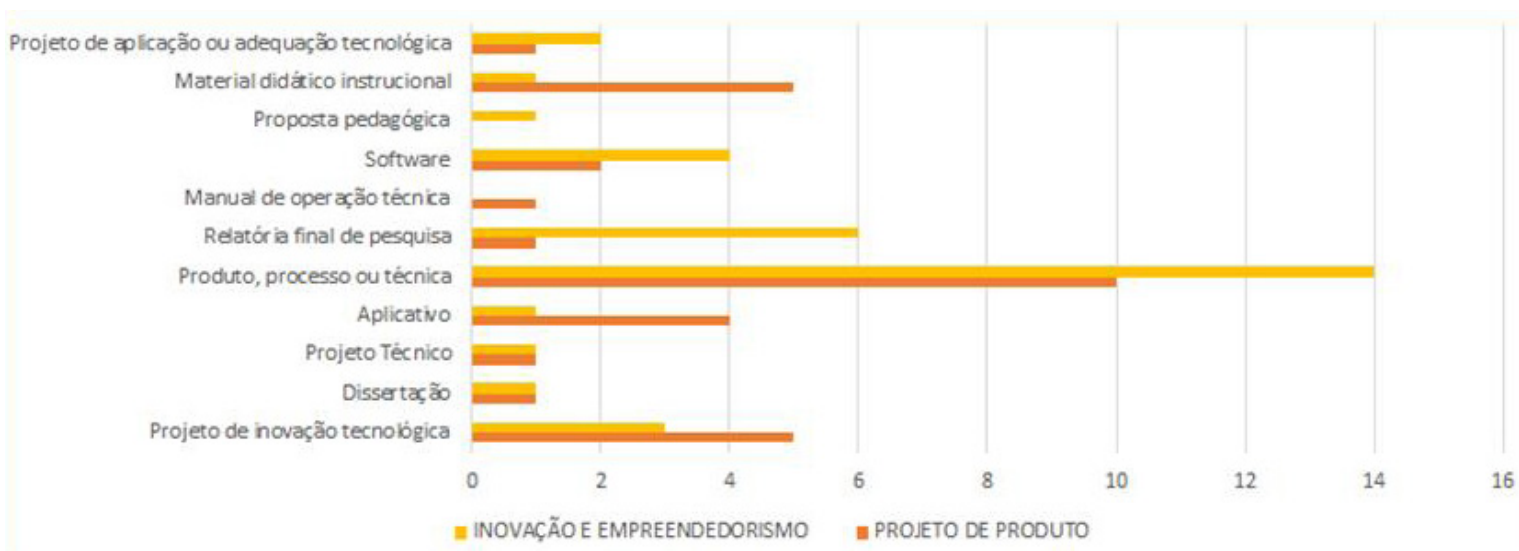

Fig 3. Produtos técnico-tecnológicos 2017-2019 Fonte: PPG-DTI (2020)
Nesse período, os resultados advém de inúmeras ações planejadas pelo Programa e das práticas de ensino e pesquisa no âmbito das disciplinas. 


\section{Linha do tempo do PPG-DTI}

No decorrer de 2015 a 2019, o PPG-DTI foram realizadas diversas ações e articulações, interna e externa, de forma democrática, tendo como referência o Documento da Área de Arquitetura, Urbanismo e Design, de modo ampliar a inserção do Programa na sociedade.

Sejam essas ações por meio dos produtos técnicos-tecnológicos oriundos dos grupos de pesquisa, projeto institucional ou projeto interinstitucional que fortalecem a área e fundamentalmente agem na formação de competências dos egressos, mas também pela socialização do conhecimento, em conjunto com outros parceiros, docentes de Instituições nacionais e internacionais, assim como de docentes e coordenadores de Programas vinculados à área do PPG-DTI.

\begin{tabular}{|c|c|}
\hline ANO & PRINCIPAIS AÇÕES \\
\hline \multirow{3}{*}{2015} & $\begin{array}{l}\text { Abertura do } 1^{\circ} \text {. Edital do PPG-DTI com } 17 \text { alunos regulares e } 1 \text { alu- } \\
\text { no em caráter especial e aulas iniciadas no mês de agosto. }\end{array}$ \\
\hline & $\begin{array}{l}\text { Realizou-se a V Mostra de Pós Graduação, em conjunto com o V Congresso Integrado do Co- } \\
\text { nhecimento e XII Encontro de Iniciação Científica e II Mostra de Extensão, totalizaram } 370 \\
\text { trabalhos e } 20 \text { Instituições de Ensino Superior, além da participação de } 118 \text { bolsistas. }\end{array}$ \\
\hline & $\begin{array}{l}\text { No mês de novembro é realizada a "Semana do Design } 2015 \text { ", tendo como principal palestrante } 0 \\
\text { Prof. Dr. Ricardo Triska, Coordenador da Área de Arquitetura, Urbanismo e Design - AUD / CAPES }\end{array}$ \\
\hline \multirow{3}{*}{2016} & $\begin{array}{l}\text { É organizado o Encontro de Extensão e Pós-Graduação - Vale do Paraíba, Sul Flu- } \\
\text { minense e Sul de Minas com a participação de Instituições EEL-USP e FATEC }\end{array}$ \\
\hline & $\begin{array}{l}\text { Participação de docentes e alunos na apresentação e trabalhos no I Congresso Interna- } \\
\text { cional / VII Workshop: Design \& Materiais 2016, evento organizado pelo PPG Design - Es- } \\
\text { cola de Artes, Arquitetura, Design e Moda da Universidade Anhembi Morumbi - UAM. }\end{array}$ \\
\hline & $\begin{array}{l}\text { Nos dias de } 10 \text { a } 12 \text { de novembro realiza-se o XIII Encontro de Iniciação Científica, XI Mostra } \\
\text { de Pós-Graduação e a III Mostra de Extensão do Centro Universitário Teresa D’Ávila - UNI- } \\
\text { FATEA com o tema "Produção da ciência em rede: perspectivas e desafios", referindo-se à } \\
\text { formação das REDES como elos interinstitucionais, internacionais e interdisciplinares. }\end{array}$ \\
\hline
\end{tabular}


Participação de docentes e discentes com trabalhos apresentados no II Congresso Internacional - VIII Workshop: Design \& Materiais 2017, realizado no período de 11 a 14 de junho, em Joinville-SC, na Universidade da Região de Joinville (Univille).

2017 Firma-se convênios/acordos de cooperação institucionais com Instituto Politécnico de Leiria (Portugal); Universidad Juárez Autónoma de Tabasco (México); Universidade Católica Portuguesa (Portugal); Universidade do Minho (UM); Universidade do Porto (UP); Universidade de Coimbra (UC); Universidade Técnica de Lisboa (UTL); Escola Superior de Artes e Design (ESAD) Matosinhos e com a Universidad de Matanzas (Cuba).

No mês de outubro aconteceu o XIV Encontro de Iniciação Cientííca, XII Mostra de Pós-Graduação e IV Mostra de Extensão, contando com a participação dos discentes e docentes do PPG-DTI.

Em março a Pró-Reitoria de Pesquisa cria o Núcleo de Inovação Tecnológica - NIT, nomeando o Prof. Dr. Nelson Tavares Matias como coordenador, passando a atuar na estruturação e planejamento, com enfoque no Manual Procedimental de Propriedade Intelectual do NIT.

No mês de agosto, o PPG promoveu a Aula Magna realizada pelo Prof. Dr. Wilson Ribeiro dos Santos Junior, Coordenador da Área de Arquitetura, Urbanismo e Design - AUD/ CAPES, intitulada "Desafios Urbanos no Século XXI: a re-significação dos espações públicos das grandes cidades".

Realizam-se os XV Encontro de Iniciação Científica, XIII Mostra de Pós-Graduação e V Mostra de Extensão. 0 evento contou o total de 245 trabalhos recebidos e 212 trabalhos aprovados. Os autores estiveram representando 50 Instituições entre Universidades (Unesp-Guaratinguetá, USP-Lorena, UNIFEI-Itajubá, UERJ - Resende, UNITAU - Taubaté, UNIVAP (São José dos Campos), FATEC/ ETEC, COTEL/USP, Faculdade de Enfermagem Wenceslau Braz (Itajubá) e Empresas (Tecnoval, Valfilm, lochpe-Maxion, Santa Casa de Misericórdia de Aparecida, de Lorena e de Guaratinguetá)

É promovido I Seminário do Programa de Pós-Graduação em Design, Tecnologia e Inovação. Foram apresentados 34 pôsteres e selecionados 43 resumos, de autoria de alunos e professores de Programas de Pós-Graduações da UNESP - Campus Bauru, Universidade Federal de Itajubá/ MG (UNIFEI), Universidade Federal Rural do Rio de Janeiro (UFRRJ), Universidade Federal de Santa Catarina (UFSC), Pontifícia Universidade Católica de São Paulo - PUC/SP e do UNIFATEA. 
Abertura do $1^{\circ}$ Semestre letivo de $2019 \mathrm{com}$ a aula magna proferida pelo Prof. Dr. Eugenio Andrés Díaz Merino intitulada "Instrumentação Tecnológica Aplicada ao design, engenharia e saúde".

Realização do $1^{\circ}$. Encontro de Grupos de Pesquisa do PPG-DTI das duas linhas de pesquisa do Programa: Projeto de Produto e em Inovação e Empreendedorismo

Organiza o III Congresso Internacional e o IX Workshop de Design e Materiais 2019, com

20194 sessões temáticas (Materiais experimentais, Materiais sustentáveis, Materiais inovadores e Materiais tradicionais) e sessão temática para apresentação de pesquisas do Ensino Médio, totalizando 47 trabalhos apresentados, 35 Instituições, incluindo universidades nacionais e estrangeiras, e cerca de 300 pessoas entre pesquisadores e participantes.

Criação do grupo Alumini com 17 participantes

No mês de novembro ocorreu a XIV Mostra de Pós-Graduação, juntamente com o XVI Encontro de Iniciação Científica, VI Mostra de Extensão e o II Seminário do Programa de Pós-Graduação em Design, Tecnologia e Inovação - PPG-DTI.

As ações têm proporcionado o desenvolvimento do Programa e a sua percepção na região e fora dela, favorecendo a sua demanda. Historicamente, no PPG-DTI, a demanda de interessados e de inscritos sempre superou as 15 vagas oferecidas em cada semestre e, de modo geral, os confirmados também superaram essa quantidade. Em 2015, ano em que iniciou a $1^{\text {a }}$ turma, foram 29 interessados e inscritos e 23 confirmaram participação no Processo Seletivo, mas somente num único semestre.

No segundo ano, em 2016, houveram 63 interessados e 55 inscritos. Comparativamente, em 2019, o número de interessados foi superior, com total de 69 interessados e o número de inscritos foi de 41 candidatos. $\mathrm{Na}$ média do período de 2015 a 2019, verificou-se a quantidade de 26,4 interessados e 26,8 inscritos.

No tocante ao número de confirmados nos Editais em cada semestre, verifica-se, que no $1^{\circ}$. Edital, houveram 23 candidatos que confirmaram a participação no Processo Seletivo. Também pode-se verificar que no período de 2015 a 2019, a média de inscritos foi de 20,1 por semestre, ou seja, superior às 15 vagas normalmente ofertadas no semestre, Tabela 1.

Ressalta-se que a Instituição é privada e, portanto, trata-se de ingressantes pagantes, embora sejam ofertadas oportunidades de bolsa institucional e descontos de empresa conveniada. Há de se destacar que a demanda do Curso é superior ao número desejável de 15 alunos matriculados no semestre. 
Tabela 1. Demanda versus oferta de vagas no período de 2015 - 2019 Fonte: PPG-DTI (2020)

\begin{tabular}{|c|c|c|c|c|c|c|}
\hline Ano & Interessados & Inscritos & & $\%$ Confirmados & & $\%$ \\
\hline 2015.2 & 29 & $12 \%$ & 29 & $12 \%$ & 23 & $13 \%$ \\
\hline 2016.1 & 37 & $16 \%$ & 29 & $12 \%$ & 23 & $13 \%$ \\
\hline 2016.2 & 26 & $11 \%$ & 26 & $11 \%$ & 23 & $13 \%$ \\
\hline 2017.1 & 7 & $3 \%$ & 23 & $10 \%$ & 18 & $10 \%$ \\
\hline 2017.2 & 29 & $12 \%$ & 31 & $13 \%$ & 21 & $12 \%$ \\
\hline 2018.1 + VR & 21 & $9 \%$ & 40 & $17 \%$ & 28 & $15 \%$ \\
\hline $2018.2+V R$ & 20 & $8 \%$ & 22 & $9 \%$ & 14 & $8 \%$ \\
\hline 2019.1 & 30 & $13 \%$ & 26 & $11 \%$ & 17 & $9 \%$ \\
\hline 2019.2 & 39 & $16 \%$ & 15 & $6 \%$ & 14 & $8 \%$ \\
\hline Totais & 238 & $100 \%$ & 241 & $100 \%$ & 181 & $100 \%$ \\
\hline MD Geral & 26,4 & $11 \%$ & 26,8 & $11 \%$ & 20,1 & $11 \%$ \\
\hline
\end{tabular}

Contudo, observa-se que após o segundo semestre de 2019, o Brasil e todo o mundo foi abalado pela pandemia da Covid-19, com efeitos ainda não previsíveis nas dimensões econômicas e sociais.

O recorte histórico do Programa no período de 2015 a 2019 permite demonstrar com segurança que as práticas adotadas têm gerado efeitos positivos para o avanço do conhecimento na subárea do Design e que possibilitam a formação de habilidades e competências dos egressos.

Cabe destacar as atividades de ensino e de extensão promovidas pelas disciplinas e projetos de pesquisa.

\section{Reflexos do PPG-DTI na produção científica regional e suas contribuições para o desenvolvimento do Design}

A contemporaneidade sugere que os indivíduos vivam em sociedade e aprendam de forma dinâmica e veloz, apoiados nas mais diversas experiências e imersos em cenários constituídos por computadores, aparatos eletrônicos e tecnologias dos mais variados tipos e fins que requerem manutenção, atualização e renovação numa celeridade muito grande, o que define, portanto, a velocidade de aprendizagem e desenvolvimento, como características primordiais para que os indivíduos se insiram e atendam a essas necessidades de uma sociedade em constante mutação.

No curso desse cenário, a pós-graduação stricto sensu tem se consolidado como uma das experiências mais exitosas da política educacional brasileira, reconhecida por sua qualidade pela comunidade nacional e internacional, configurando-se uma importante mola propulsora do desenvolvimento nacional, à proporção que se revela como o setor responsável pela formação de recursos humanos de alto nível, constituindo-se numa das bases do Sistema Nacional de Ciência e Tecnologia e como setor estratégico no âmbito das políticas de educação. 
Note-se que essas novas configurações sociais de desenvolvimento científico e tecnológico passam a sustentar também um diálogo mais estreito entre educação e mercado como forma de assimilar os parâmetros deste desenvolvimento como modelo para a criação de novas políticas científicas e educativas. É na efervescência dessas configurações que se alicerçam os fundamentos que orientam a criação dos mestrados profissionais. Conforme prevê a Portaria no 80, de 16 de dezembro de 1998 (BRASIL, 1998) o mestrado profissional tem em sua articulação curricular a ênfase em estudos e técnicas diretamente voltadas ao desempenho de um alto nível de qualificação profissional, constituindo-se esta como uma única ponta de diferenciação em relação ao mestrado acadêmico. Como aponta a própria CAPES (Coordenação de Aperfeiçoamento de Pessoal de Nível Superior):

\footnotetext{
Antes de mais nada, o mestrado profissional (MP) é um título terminal, que se distingue do acadêmico porque este último prepara um pesquisador, que deverá continuar sua carreira com o doutorado, enquanto no MP o que se pretende é imergir um pós-graduando na pesquisa, fazer que ele a conheça bem, mas não necessariamente que ele depois continue a pesquisar. O que importa é que ele (1) conheça por experiência própria o que é pesquisar, (2) saiba onde localizar, no futuro, a pesquisa que interesse a sua profissão, (3) aprenda como incluir a pesquisa existente e a futura no seu trabalho profissional. Nada disso é trivial. o terceiro ponto é, por sinal, razoavelmente difícil. Por isso, o MP não pode ser entendido como um mestrado facilitado (CAPES, 2007).
}

A gênese do PPG-DTI pressupõe a participação de setores diferentes e sua construção, por conseguinte, torna-se coletiva e social: coletiva porque envolve uma equipe de pessoas trabalhando em prol de um ou vários objetivos, e social porque inclui diferentes visões de mundo, com seus respectivos objetivos e interesses.

Ferreira (2014) observa que a implementação dos mestrados profissionais, a partir do final dos anos de 1990 e nos anos da década seguinte, passa a ser tratada como uma política educacional, independente das muitas críticas formuladas pelo meio acadêmico a essa modalidade. Dessa forma, a Capes tem induzido fortemente a oferta de mestrados profissionais em todas as áreas de conhecimento, por meio dos editais para apresentação de cursos novos.

Ainda com embasamento em Ferreira (2014), pode-se afirmar que os documentos da Capes evidenciam que os mestrados profissionais não devem ter ênfase na pesquisa científica já que os perfis profissionais esperados para esses cursos são de profissionais que já estão inseridos no mercado de trabalho, um dos pontos principais para o qual convergem as resistências a essa modalidade de pós-graduação. 
Nesse sentido, as ações desenvolvidas no âmbito do PPG- DTI revelam um conjunto de propostas em que indivíduos, sociedade, poder público e iniciativa privada interagem conjuntamente, a fim de oportunizar a formação de agentes locais de inovação, que no cerne da pesquisa aplicada reflitam sobre as práticas inovativas, considerando-se o ambiente corporativo como um espaço para o desenvolvimento de projetos para melhoria de processos, dispositivos/componentes, produtos em consonância com as linhas de pesquisa do Programa, bem como perfil do corpo docente e dos ingressantes.

O desafio que estudantes e professores enfrentam está justamente no movimento de aproximação entre Universidade, Empresas e Sociedade e no estabelecimento de tema emergentes, como: Smart cities, Tecnologia Assistiva, Design de Serviços, Novos Materiais, Energias Renováveis, Prototipagem Rápida, Inteligência Artificial (AI), Realidade Aumentada e Virtual, Inovação e Tecnologias Sociais (TS), dentre outros, que permitam identificar canais para intensificação do diálogo interdisciplinar, para trocas de experiências entre programas e para divulgação do conhecimento interdisciplinar gerado.

A atuação do PPG-DTI como espaço de ensino de pós-graduação é referência do saber científico e bastante importante para a comunidade regional, pois tem capacitado fatores humanos para a pesquisa, o ensino, a extensão e o para o exercício profissional especializado, contribuindo para a melhoria da qualidade de vida e do desempenho de instituições públicas e privadas, ao marcar a posição competitiva das organizações, além de prover a melhoria das competências acadêmicas, na exploração do saber universal.

As pesquisas desenvolvidas lograram estimular projetos que surgiram no âmbito dos cursos de graduação ou de pós, dando preferência aos que se apoiaram em diagnósticos da realidade local e regional, e que puderam se abrir a contextos mais amplos.

Isso marca a importância do incentivo direto do PPG-DTI à pesquisa, que por meio de ações concretas, que aproximou estudantes (graduação e de pós-graduação e mesmo de nível médio da rede oficial de ensino) e docentes do UNIFATEA e de instituições congêneres, em movimentos de socialização da produção acadêmica, tais como fóruns regionais; jornadas pedagógicas; simpósios técnico-profissionais; mostras de projetos de pós-graduação e encontros de iniciação científica.

Esses movimentos estimularam parcerias, com instituições nacionais e estrangeiras, propiciadoras do enriquecimento do senso crítico de nossas produções e do discernimento para a escolha e a definição de linhas de investigação, como também de metodologias e desenvolvimento da pesquisa, e, por isso, celebradas para projetos e ações culturais e tecnocientíficas conjuntos.

Os docentes do PPG-DTI mantem contatos frequentes com a maioria dos seus ex-orientandos, facilitando o levantamento de informações dos egressos do Programa, e normalmente os contatos são mantidos por meio das redes sociais (Linkedin e Facebook), e-mail ou grupos no whatsapp. 
No período de 2015 (segundo semestre) a 2019, o Programa contribuiu por meio da cooperação e da interlocução das atividades planejadas de ensino, pesquisa e extensão, formar 65 egressos, considerando-se que a formação na graduação tem por característica a heterogeneidade. Conforme acompanhamento dos egressos contata-se que houve ganhos de oportunidades profissionais, Quadro 2.

Quadro 2. Síntese dos relatos das oportunidades dos egressos do PPG-DTI Fonte: PPG-DTI (2020)

\section{Estratégias de Acompanhamento e ações de interlocução com o merca- do ofertado pelo PPG DTI nas realizações profissionais do egressos.}

\begin{tabular}{|l|l|l|}
\hline Assumiu coordenação & $\begin{array}{l}\text { Ingresso em progra- } \\
\text { ma de doutorado }\end{array}$ & $\begin{array}{l}\text { Ingresso em programa de } \\
\text { mestrado na Universidade } \\
\text { Católica de Portugal }\end{array}$ \\
\hline $\begin{array}{l}\text { Assumiu cargo de Diretor } \\
\text { em Secretaria da Saúde }\end{array}$ & $\begin{array}{l}\text { Teve o projeto de mestrado } \\
\text { incubado no Pq Tecnológico } \\
\text { de São José dos Campos }\end{array}$ & $\begin{array}{l}\text { Assumiu a coordenação } \\
\text { da graduação em Admi- } \\
\text { nistração no UNIFATEA }\end{array}$ \\
\hline $\begin{array}{l}\text { Contrata como coordena- } \\
\text { dora na Editora Santuário } \\
\text { Nacional de Aparecida }\end{array}$ & $\begin{array}{l}\text { Obteve evolução funcional em } \\
\text { instituição de ensino pública }\end{array}$ & $\begin{array}{l}\text { Ingresso no Instituto } \\
\text { Nacional de Pesquisas } \\
\text { Espaciais - INPE }\end{array}$ \\
\hline $\begin{array}{l}\text { Ingresso como docente em } \\
\text { Instituição de nível superior }\end{array}$ & Assumiu cargo de coordenação \\
$\begin{array}{l}\text { Convites para pales- } \\
\text { tras e seminários }\end{array}$ & $\begin{array}{l}\text { Ingresso como docente } \\
\text { em curso técnico }\end{array}$ \\
\hline Transformação da dis- & $\begin{array}{l}\text { Alavancou oportu- } \\
\text { nidades na gestão } \\
\text { pública municipal }\end{array}$ \\
\hline
\end{tabular}

As reflexões diante das oportunidades alcançadas por mais de um egresso, alguns dos quais participam como docentes nos cursos lato sensu da própria instituição.

As interlocuções e as pesquisas que se desenvolvem no PPG-DTI apontam para a necessidade de se pensar a pesquisa em design como um movimento que envolve uma dimensão coletiva e que articule uma dinâmica de desejos e interesses, por meio da qual se possa extrair soluções que atendam comunidades interessadas e atuantes visando a construção de uma inteligência coletiva.

A questão que aqui se coloca é: como os conhecimentos produzidos pelas pesquisas se constituem e se validam como respostas para as comunidades/atores/sujeitos envolvidos no processo? 
A pesquisa enquanto processo sistemático de construção do conhecimento para o design tem como metas principais gerar novos conhecimentos e/ou corroborar ou refutar algum conhecimento pré-existente. Produzir, nesse contexto, é um processo de aprendizagem tanto do indivíduo que a realiza quanto da sociedade na qual ela se desenvolve.

Partindo-se da premissa de que o conhecimento não é matéria ou assunto acabado, é infinito e deve ser continuamente explorado, repensado e reformulado, as pesquisas orientadas no PPG-DTI articulam-se na obtenção de informações, de compreensão das certezas e dos direcionamentos necessários para conceituar e projetar produtos e serviços de forma coerente com as necessidades dos usuários. Isso significa dizer que essas pesquisas servem como possibilidade para que nos laboratórios do PPG-DTI os pesquisadores possam exercer seu papel criativo para transformar os dados coletados em soluções palpáveis, eficientes e que tragam resultados no desenvolvimento das comunidades envolvidas.

As ações de pesquisa desenvolvidas no PPG-DTI configuram-se em espaços de confronto dialético entre teoria e prática, buscando chamar a atenção para as variáveis existentes nas situações em que participam designers e não-designers. Essas pesquisas surgem, assim, como possibilitadas de um movimento de mutualidade recíproca na compreensão de si, do outro, do contexto particular e geral, do mundo e, dessa forma de transformação de contextos como resultado de um processo de conscientização sobre a própria prática e a do outro, geralmente aliada ao aprofundamento teórico e crítico que expande os processos de produção dos projetos, produtos e serviços.

\section{Considerações Finais}

O PPG-DTI tem por princípio fundamental a sua missão de promover a formação de profissionais competentes para produzir e aplicar conhecimentos e tecnologias no contexto da sua área de concentração. Os componentes curriculares são desenvolvidos com base nos objetivos de formação do egresso, pautados pela compreensão e análise crítica do conhecimento, indissociáveis do contexto da sociedade. 0 aprofundamento do conhecimento articula-se com a pesquisa, desdobrando atividades de modo interdisciplinar e multidisciplinar, e procurando viabilizar aplicações para diferentes segmentos da sociedade. No percurso do Programa, desde 2015, ocorrerão diversas ações com a aproximação da sociedade, tais como: os encontros de iniciação de iniciação científica; as mostras de pós-graduação; workshops nas empresas e com profissionais em nossos laboratórios; visitas técnicas e seminários proferidos por profissionais e projetos de pesquisa com a participação das empresas. Destacam-se também os minicursos como atividades planejadas de projetos de extensão são promovidos por docentes e discentes do Programa e com a participação do convidados. 
Design, Reflexões e Experiências: contribuições de um Programa de Mestrado Profissional para o avanço do conhecimento em Design

\section{Referências}

ARQUITETURA, URBANISMO E DESIGN - AUD. In: CAPES. 2020. Documento de Área 2019. Disponível em: https://www.gov.br/capes/pt-br/centrais-de-conteudo/documento-area-aud-pdf.

CAPES - Coordenação de Aperfeiçoamento de Pessoal de Nível. Ministério da Educação. O que é mestrado profissional. 2007. Disponível em: https://www.gov.br/capes/pt-br/centrais-de-conteudo/ApresentacaoJosealdoTonholo1.pdf. Acessado em: 05 fev 2021.

FERREIRA, Diógenes Arruda. 0 processo de criação e institucionalização de mestrado profissional com foco em educação no nordeste do brasil. Dissertação de Mestrado. Universidade Federal de Pernambuco: Recife, 2014.

PPG-DTI - Programa de Mestrado Profissional em Design, Tecnologia e Inovação. Relatórios de gestão. Lorena, 2019.

PPG-DTI - Programa de Mestrado Profissional em Design, Tecnologia e Inovação. Relatórios de gestão. Lorena, 2020. 